"This document is the Accepted Manuscript version of a Published Work that appeared in final form in Nano Letters, copyright (C) American Chemical Society after peer review and technical editing by the publisher. To access the final edited and published work see:

http://dx.doi.org/10.1021/acs.nanolett.6b04936." 


\title{
Electrical and thermal transport in coplanar polycrystalline graphene-hBN heterostructures
}

\author{
J.E. Barrios Vargas, ${ }^{* \dagger}$ B. Mortazavi, ${ }^{\ddagger}$ A.W. Cummings, ${ }^{\dagger}$ R. Martinez-Gordillo, \\ M. Pruneda, ${ }^{\dagger}$ L. Colombo, ${ }^{\dagger, \delta}$ T. Rabczuk, and S. Roche ${ }^{*,+, \|}$ \\ $\dagger$ Catalan Institute of Nanoscience and Nanotechnology (ICN2), CSIC and The Barcelona \\ Institute of Science and Technology, Campus UAB, 08193 Barcelona, Spain \\ $\ddagger$ Institute of Structural Mechanics, Bauhaus-Universität Weimar, Marienstr. 15, D-99423 \\ Weimar, Germany \\ - CINaM - Centre Interdisciplinaire de Nanoscience de Marseille, France \\ $\S$ Dipartimento di Fisica, Universit di Cagliari, Cittadella Universitaria, I-09042 \\ Monserrato ( $\mathrm{Ca}$ ), Italy \\ |ICREA - Institució Catalana de Recerca i Estudis Avançats, 08010 Barcelona, Spain \\ E-mail: jose.barrios@icn2.cat; stephan.roche@icn2.cat
}

\section{Abstract}

We present a theoretical study of electronic and thermal transport in polycrystalline heterostructures combining graphene $(\mathrm{G})$ and hexagonal boron nitride $(\mathrm{hBN})$ grains of varying size and distribution. By increasing the $\mathrm{hBN}$ grain density from a few percent to $100 \%$, the system evolves from a good conductor to an insulator, with the mobility dropping by orders of magnitude and the sheet resistance reaching the $\mathrm{M} \Omega$ regime. The Seebeck coefficient is suppressed above $40 \%$ mixing, while the thermal conductivity of polycrystalline $\mathrm{hBN}$ is found to be on the order of $30-120 \mathrm{Wm}^{-1} \mathrm{~K}^{-1}$. These results, agreeing with available experimental data, provide guidelines for tuning G-hBN properties in the context of two-dimensional materials engineering. In particular, while we proved that both electrical and thermal properties are largely affected by morphological features (like e.g. by the grain size and composition), we find in all cases that nm-sized polycrystalline G-hBN heterostructures are not good thermoelectric materials.

Introduction. Owing to a small lattice mismatch $(2 \%)$, graphene and hexagonal boron nitride can be assembled in coplanar twodimensional heterostructures ${ }^{(1)}$. Such atomic sheets, covering a wide range of compositions, result in new materials with properties complementary to those of graphene and hBN, such as tunable bandgap optoelectronic materials ${ }^{(2)}$. Graphene is well appreciated for its high electrical ${ }^{(3)}$ and thermal conductivities ${ }^{(4)}$, whereas $\mathrm{hBN}$ is an electrical insulator with to date an unmeasured thermal conductivity ${ }^{(5,6)}$. Large-scale coplanar G-hBN heterostructures have been successfully fabricated using chemical vapor deposition (CVD), enabling the possible control of periodic arrangements of domains whose sizes range from tens of nanometers to millimeters ${ }^{(\gamma-10)}$. Their charge transport properties can be, however, quite surprising, such as the prescence of a metal-insulator transition ${ }^{(11-13)}$ and anomalous transport phenomena, which are not fully understood. ${ }^{(14)}$ Additionally, fast CVD growth results in polycrystalline materials with grains of varying sizes and morphologies, and the electronic and thermal properties of these materials are limited by the presence of grain boundaries $(\mathrm{GBs})^{(15-19)}$.

In polycrystalline graphene, GBs are characterized by Van Hove singularities near the Dirac 
point ${ }^{(20-22)}$, whereas in $\mathrm{hBN}$ the GBs reduce the bandgap and introduce gap states generated by the presence of $\mathrm{B}-\mathrm{B}$ or N-N bonds ${ }^{(23)}$. The interface between $\mathrm{G}$ and $\mathrm{hBN}$ is also expected to give rise to local boundary states, especially at low energies ${ }^{(24,25)}$. GBs are also usually accompanied by local structural deformation, which enhances phonon scattering and thus lowers thermal conduction. The thermal properties of polycrystalline graphene have been theoretically calculated using molecular dynamics simulations as a function of average grain size ${ }^{(17,18,26,27)}$, in fair agreement with experimental results ${ }^{(4)}$.

Recently, a sample of CVD-grown graphene was gradually converted into $\mathrm{hBN}$, and it was observed that chemical substitutions are initiated around structural defects. This process of conversion demonstrated a fine tunability between highly conductive graphene and insulating $\mathrm{hBN}^{(13)}$. To date however, the electronic and thermal properties of CVD-grown hybridized G-hBN heterostructures are poorly understood, and their potential use in energy harvesting, optoelectronic, or nanoelectronic applications remains unclear.

Here we use quantum transport and molecular dynamics (MD) simulations to calculate the electronic and thermal properties of polycrystalline G-hBN heterostructures with varying grain size and distribution. The electronic mobility and sheet resistance are studied as a function of the density of hBN grains, which ranges from a few percent to full coverage. The contribution of GB interface states to the transport properties is also illustrated and quantified. By performing a complete calculation of thermal and electrical transport, we estimate the thermoelectric conversion ratio and find that it remains far too low to be useful for energy harvesting applications.

Generation of samples. Polycrystalline G-hBN heterostructures with uniform average grain size were generated using a Voronoi algorithm, resulting in large square periodic samples containing up to 3 million atoms ${ }^{(17,28)}$. The algorithm starts with a random selection of nucleation centers within a square cell of predefined dimension, which dictates the aver- age grain size as $L_{\text {grain }}=\sqrt{L^{2} / n_{\text {grains }}}$, where $L$ is the sample length and $n_{\text {grains }}$ is the number of grains. Next we set a random crystal orientation for each nucleation site and we use a Voronoi method to construct the grains. The atoms along the GBs with separation below $0.1 \mathrm{~nm}$ are removed, and an MD annealing process is used to construct the GBs, setting all the atoms as carbon. We use the LAMMPS simulation package ${ }^{(29)}$, the second-generation reactive empirical bond order potential ${ }^{(30)}$, and a small time increment of $0.1 \mathrm{fs}$. The annealing starts with a 3 -ps equilibration at room temperature using the Nosé-Hoover thermostat, continues with a heating up to $3000 \mathrm{~K}$ for $12 \mathrm{ps}$ and keeping this temperature for $3 \mathrm{ps}$, and ends with a cooling back down to room temperature for 10 ps. Finally, based the concentration of $\mathrm{hBN}$, we assign which grains are graphene and which ones are hBN (Fig. 1).

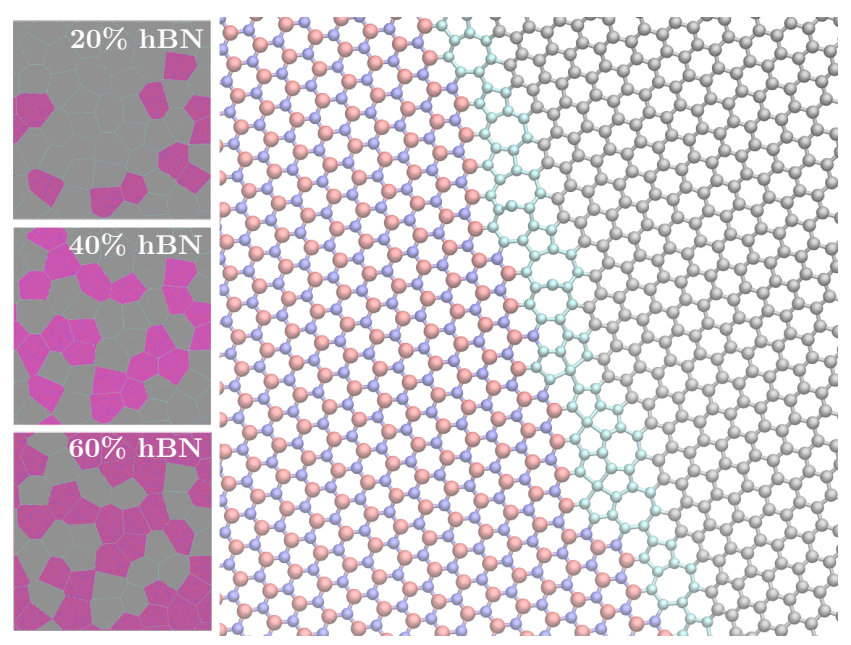

Figure 1: Left panel: square periodic polycrystalline structures with three different concentrations of hBN $(20 \%, 40 \%$ and $60 \%)$. Right panel: magnification of the polycrystalline structure showing a typical interface between graphene and hBN grains.

Electronic properties. We describe the electronic properties of the G-hBN heterostructures with a tight-binding Hamiltonian

$$
\mathcal{H}=\sum_{\mathbf{r}_{i}} \varepsilon_{i}\left(\mathbf{r}_{i}\right)\left|\mathbf{r}_{i}\right\rangle\left\langle\mathbf{r}_{i}\left|+\sum_{\left\langle\mathbf{r}_{i}, \mathbf{r}_{j}\right\rangle} t_{i, j}\right| \mathbf{r}_{i}\right\rangle\left\langle\mathbf{r}_{j}\right|,
$$

where $\varepsilon_{i}\left(\mathbf{r}_{i}\right)$ is the on-site potential of each atom 
and $t_{i, j}$ is the hopping between nearest neighbors. In systems containing 1D interfaces between two different 2D materials, the electronic properties are sensitive to the interface termination, and thus care must be taken when describing the GBs between graphene and hBN grains. For example, zigzag BN nanoribbons are polar, presenting bound charge of opposite signs at the B and $\mathrm{N}$ edges. In hybrid systems, mobile electrons from the graphene will tend to screen the excess interfacial charge, which changes the potential profile across the GB. Therefore, we modify the on-site term of the Hamiltonian to include a position-dependent electrostatic potential, which can be derived from the screened Poisson equation considering point charges midway between the C-B or C-N interfacial bonds. The on-site term of the TB Hamiltonian can then be written as ${ }^{(31)}$

$$
\begin{aligned}
\varepsilon_{i}\left(\mathbf{r}_{i}\right)=\varepsilon_{i 0} & +\sum_{\alpha}^{n_{q}} \frac{A_{i}^{\mathrm{B}}}{\left|\mathbf{r}_{i}-\mathbf{r}_{\alpha}^{\mathrm{B}}\right|} e^{-\frac{\left|\mathbf{r}_{i}-\mathbf{r}_{\alpha}^{\mathrm{B}}\right|}{\lambda_{i}}} \\
& -\sum_{\alpha}^{n_{q}} \frac{A_{i}^{\mathrm{N}}}{\left|\mathbf{r}_{i}-\mathbf{r}_{\alpha}^{\mathrm{N}}\right|} e^{-\frac{\left|\mathbf{r}_{i}-\mathbf{r}_{\alpha}^{\mathrm{N}}\right|}{\lambda_{i}}},
\end{aligned}
$$

where $\varepsilon_{i}\left(\mathbf{r}_{i}\right)$ denotes the on-site energy for an atom of type $i$ (either carbon, boron, or nitrogen) at position $\mathbf{r}_{i}, \varepsilon_{i 0}$ is the on-site energy of atoms far from the GBs, $A_{i}^{\mathrm{B}}\left(A_{i}^{\mathrm{N}}\right)$ is the strength of the potential arising from the $\mathrm{C}-\mathrm{B}$ $(\mathrm{C}-\mathrm{N})$ interface, $\mathbf{r}_{\alpha}^{\mathrm{B}}\left(\mathbf{r}_{\alpha}^{\mathrm{N}}\right)$ is the position of the excess charge at the C-B (C-N) interface, $\lambda_{i}$ is the decay length of the interface potential, and the sum is done for all $n_{q}$ charges within a radius of $1 \mathrm{~nm}$. The onsite potential and nearestneighbor hopping parameters have been derived from a Wannierization of DFT calculations and are given in Table 1 (see Supplementary Information for more details). Finally, because the GBs contain non-hexagonal rings, B-B or N-N bonds will be present. For these bonds we set $t_{\mathrm{BN}}$ as the hopping parameter, while the on-site energy is taken as $1.1 \varepsilon_{i 0}$.

We calculate the electronic density of states (DOS) using the Lanczos recursion method with an energy resolution of $\eta=k T=26 \mathrm{meV}$ $(T=300 \mathrm{~K})$. Figure 2(a) shows the DOS with increasing hBN grain density in steps of
Table 1: On-site and nearest-neighbor tight-

\begin{tabular}{|c|c|c|c|}
\hline \multicolumn{4}{|c|}{ On-site energy (eV) } \\
\hline$\varepsilon_{\mathrm{C} 0}$ & $\varepsilon_{\mathrm{B} 0}$ & $\varepsilon_{\mathrm{N} 0}$ & \\
\hline 0.0 & 3.09 & -1.89 & \\
\hline \multicolumn{4}{|c|}{ Boundary Electrostatic Potential parameters } \\
\hline$\lambda_{\mathrm{C}}$ & $\lambda_{\mathrm{B}}=\lambda_{\mathrm{I}}$ & $A_{i}^{\mathrm{B}}=A_{i}^{\mathrm{N}}$ & \\
\hline $6.78 \AA$ & $12.56 \AA$ & $0.56 \mathrm{eV} \cdot \AA$ & \\
\hline \multicolumn{4}{|c|}{ Nearest-neighbor hoppings (eV) } \\
\hline$t_{\mathrm{CC}}$ & $t_{\mathrm{CB}}$ & $t_{\mathrm{CN}}$ & $t_{\mathrm{BN}}$ \\
\hline-2.99 & -2.68 & -2.79 & -3.03 \\
\hline
\end{tabular}
binding Hamiltonian parameters.

$20 \%$, for an average grain size of $40 \mathrm{~nm}$. The gap is seen to progressively widen with increasing hBN concentration, but with a faster decay on the electron side of the spectrum. This electron-hole asymmetry stems from the GB states, which generate more resonances on the electron side. This can be seen more clearly for $100 \% \mathrm{hBN}$, where the formation of boundary states, with energy lying inside the gap, is illustrated by the local density of states projected over all the GB sites $\left(\operatorname{LDOS}_{\mathrm{GB}}\right.$; Fig. 2(b)). The energy resonances at -1.2 and $2 \mathrm{eV}$ have been observed experimentally, which can be associated to homoelemental bonds in the GB. (23) These states are mainly localized at the GBs, as visualized in the inset of Fig. 2(b), with stronger energy resonances on the electron side of the spectrum. The presence of such states could be at the origin of the finite electrical conductivity computed for polycrystalline hBN (see below).

We next evaluate the electronic transport properties using a real-space order-N wave packet propagation method ${ }^{(32,33)}$. The core of this method is to calculate the time-dependent diffusion coefficient as

$$
D(E, t)=\frac{\partial}{\partial t} \Delta X^{2}(E, t),
$$

where $\Delta X^{2}$ is the mean-square displacement of the wave packet

$$
\Delta X^{2}(E, t)=\frac{\operatorname{Tr}\left[\delta(E-\hat{H})|\hat{X}(t)-\hat{X}(0)|^{2}\right]}{\rho(E)}
$$



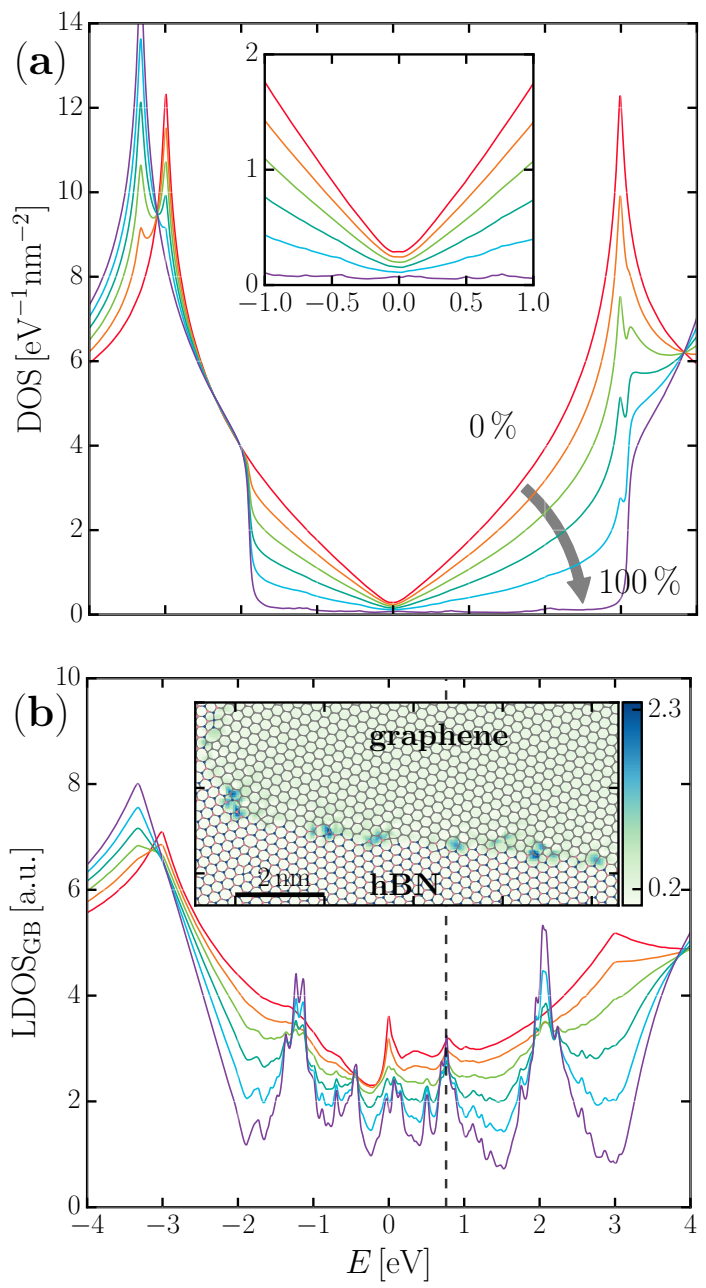

Figure 2: (a) DOS of the polycrystalline lattice with increasing hBN grain density, in steps of $20 \%$, for an average grain size of $40 \mathrm{~nm}$. Inset: magnification of the DOS in the interval $[-1,1]$ $\mathrm{eV}$. (b) $\mathrm{LDOS}_{\mathrm{GB}}$ for the same parameters. Inset: LDOS $\mathrm{GB}$ projected around a Gr-hBN interface, corresponding to the energy marked with the dashed line in the main frame.

and $\rho(E)=\operatorname{Tr}[\delta(E-\hat{H})]$ is the DOS. We evaluate the trace using the Lanczos recursion and the same parameters as the DOS. We calculate the energy-dependent semiclassical conductivity as $\sigma(E)=e^{2} \rho(E) \tilde{D}(E)$, where $\tilde{D}(E)$ is the value of the diffusion coefficient when the mean displacement has reached six times the average grain size (see Supplementary Information).

In Fig. 3(a) we report $\sigma(E)$, where a drop of more than two orders of magnitude is observed near the charge neutrality point with increasing hBN concentration. To further clarify the impact of the density of hBN grains, we fix the car- rier concentration to $n=0.3 \times 10^{12} \mathrm{~cm}^{-2}$, which is a typical value for graphene on $\mathrm{SiO}_{2}{ }^{(34)}$, and evaluate the charge mobility $\mu=\sigma(n) / n$, shown in Fig. 3(b). The sheet resistance $R$ is shown in the inset of Fig. 3(b), where one can see that the maximum value for $100 \% \mathrm{hBN}$ is about $1 \mathrm{M} \Omega$. Additionally, we estimate the GBresistivity, $\rho_{\mathrm{GB}}$, using an ohmic scaling analy$\operatorname{sis}^{(16,19)}$,

$$
R=R^{0}+\frac{\rho_{\mathrm{GB}}}{L_{\text {grain }}},
$$

where $R$ and $R^{0}$ are the sheet resistances of the polycrystalline sample and the individual grains, respectively. The estimated resistivity for the G-G interface is 0.12 and for hBN-hBN is $5.93 \mathrm{k} \Omega \cdot \mu \mathrm{m}$ (see Supplementary Information).

To complement the information about the electronic properties, we evaluate the Seebeck coefficient

$$
S(E)=-\frac{1}{|e| T} \frac{\int_{-\infty}^{\infty}\left(E^{\prime}-E\right) G\left(E^{\prime}\right)\left(-\frac{\partial f}{\partial E^{\prime}}\right) d E^{\prime}}{\int_{-\infty}^{\infty} G\left(E^{\prime}\right)\left(-\frac{\partial f}{\partial E^{\prime}}\right) d E^{\prime}},
$$

where $G$ is the sheet conductance and $f$ is the Fermi distribution. As shown in Fig. 3(c), the Seebeck coefficient of the polycrystalline samples is reduced compared to pristine graphene $^{(35)}$, but is insensitive to $\mathrm{hBN}$ concentrations below 40\%. However, beyond $40 \%$ the thermoelectric capability is strongly suppressed.

Thermal properties. In order to evaluate the thermal conductivity as a function of the grain size, we construct a finite element (FE) model in the ABAQUS package with 4000 grains constructed as Voronoi cells (right panel Figure 4(a)). Using six representative pentagon-heptagon GB structures, we extract the GB thermal conductance for G-G, G-hBN and $\mathrm{hBN}-\mathrm{hBN}$ interfaces by performing a nonequilibrium molecular dynamics (NEMD) cal- 

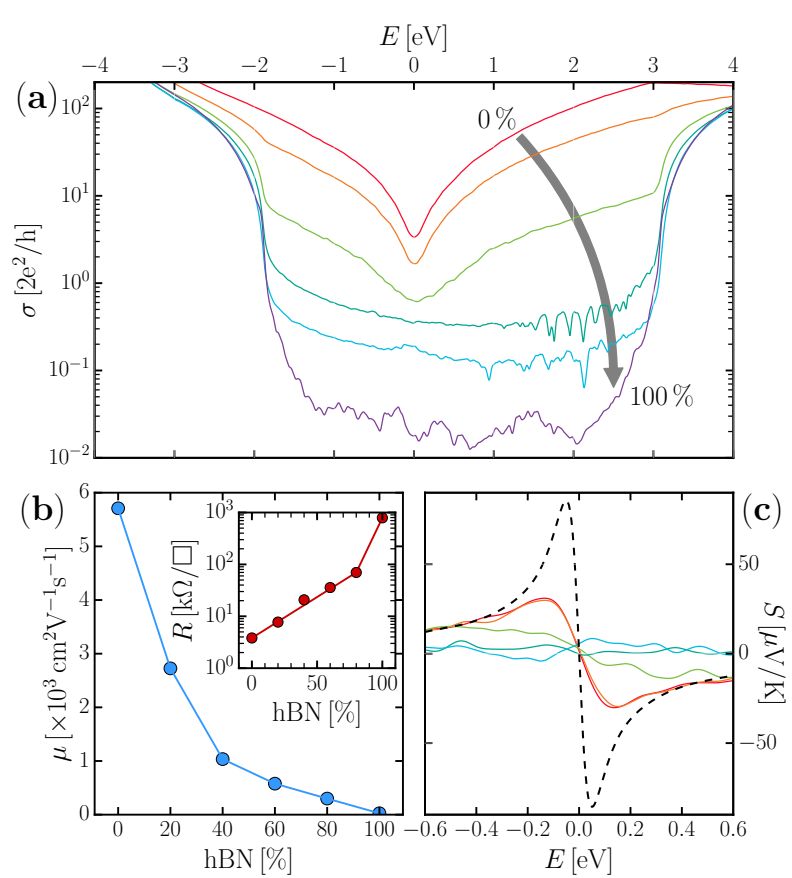

Figure 3: (a) Conductivity versus energy for various $\mathrm{hBN}$ grain densities, with an average grain size of $40 \mathrm{~nm}$. (b) Mobility as a function of hBN concentration for a fixed carrier density $n=0.3 \times 10^{12} \mathrm{~cm}^{-2}$. Inset: the sheet resistance for the same carrier density. (c) Seebeck coefficient with increasing hBN grain density, with the dashed line showing the pristine graphene value.

culation with LAMMPS (see Supplementary Information for details); which are introduced as contact conductances between interfaces. In the FE model, we include two highly conductive strips at the two ends of the structure ${ }^{(17,28)}$ and fix the ingoing (outgoing) heat flux on the left (right) side, $h_{\mathrm{f}}$. Then, we evaluate the steadystate temperature profile along the sample and use the $\Delta T$ between the strips to evaluate the effective thermal conductivity of the sample as

$$
\kappa=h_{\mathrm{f}} \frac{L}{\Delta T},
$$

where $L$ is the sample length. We calculate the themal conductivity for 16 grain sizes between 1-1000 $\mathrm{nm}$ while changing the concentration of hBN (Fig. 4(b)). The scaling of $\kappa$ shows that the impact of the GBs on thermal transport becomes negligible for grain sizes above $100 \mathrm{~nm}$. Figure 4(c) displays the thermal conductivity as a function of the hBN grain density where we observe that, for small average grain size, the minimum of thermal conductivity occurs near $70 \% \mathrm{hBN}$, similar to prior estimates ${ }^{(36)}$. This minimum can be rationalized by the fact that the thermal conductance for the G-hBN interface is lower than that of the hBN-hBN and $\mathrm{G}-\mathrm{G}$ interfaces. For larger grain sizes, where the GBs no longer dominate the thermal transport, we observe a monotonic scaling of $\kappa$ with $\mathrm{hBN}$ grain density, as the thermal conductivity of pristine $\mathrm{hBN}$ is lower than that of pristine graphene.
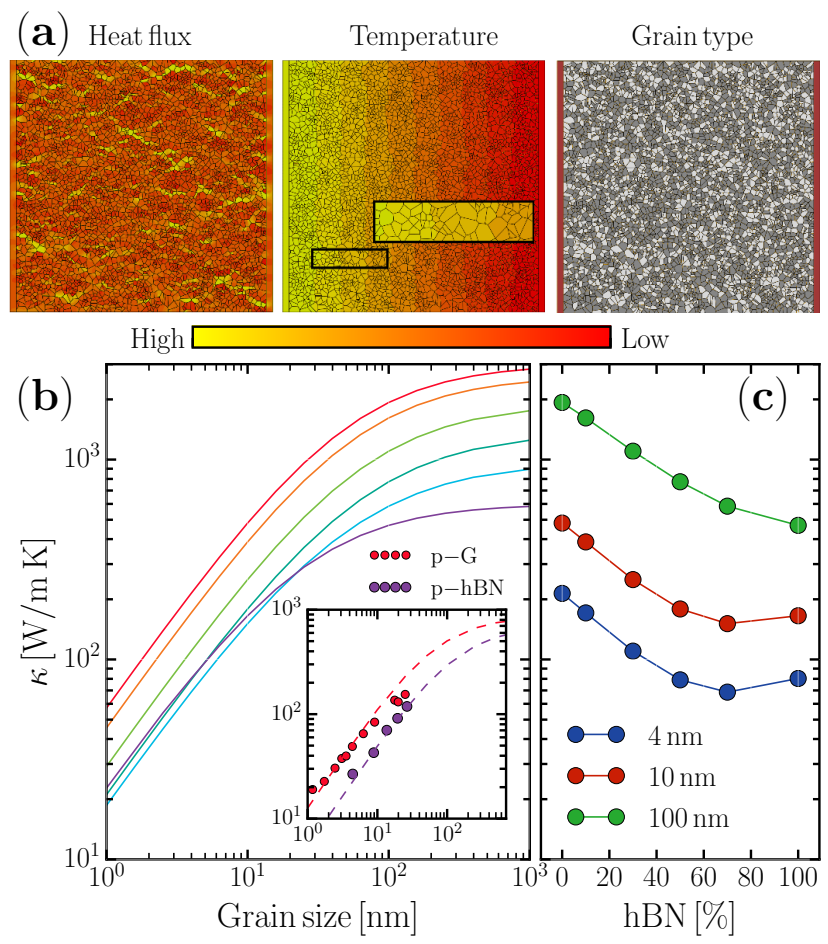

Figure 4: (a) Heat flux (left panel) and temperature profile (center panel) calculated with FE for the granular mesh shown in the right panel. (b) Thermal conductivity as a function of the average grain size calculated with FE. Inset: symbols show the polycrystalline graphene (p-G) and hBN (p-hBN) thermal conductivities calculated using AEMD, while the dashed lines show the extrapolated scaling behavior using the extracted GB conductance. (c) Thermal conductivity as a function of the hBN grain density for different average grain sizes using the FE method.

In order to validate the above $\mathrm{FE}$ analysis we perform an independent investigation based on 
MD simulations. The goal is to provide evidence that the FE analysis, although missing most of the atomic-scale details, nevertheless provides the correct gross features on thermal transport across hBN and graphene GBs. The fully atomistic study of the thermal conductivity employs an approach-to-equilibrium molecular dynamics (AEMD) method following the same approach as in Ref.(18) using the Tersoff BNC potential ${ }^{(37)}$ (see Supplementary Information). While NEMD provides direct access to the temperature drop across the GB, which is the relevant quantity needed to calculate the interface thermal resistance (and, therefore, the GB conductance), the AEMD approach is better suited to calculate the effective $\kappa$ in a large system, since it requires a comparatively smaller computational effort ${ }^{(38)}$. We observe a quantitative difference between the approach described above and AEMD, which is reflected in the extracted value of the thermal conductance of the hBN-hBN interface, $C_{\mathrm{hBN}-\mathrm{hBN}}=5.27 \mathrm{GW} / \mathrm{m}^{2} \mathrm{~K}$. From the data reported in Ref. ${ }^{(18)}$, we also estimate the thermal conductance of the G-G interface to be $C_{\mathrm{G}-\mathrm{G}}=$ $12.66 \mathrm{GW} / \mathrm{m}^{2} \mathrm{~K}$. We attribute these lower values to the structure of the GBs investigated; the GBs in the AEMD calculations tend to be disordered and meandering, as shown in Fig. 1, while the GBs used in the NEMD method were mirror symmetric and perfectly periodic arrays of pentagon-heptagon pairs. The inset of Fig. 4(b) shows the thermal conductivity of polycrystalline graphene and hBN using the FE method and the GB conductances extracted from the AEMD method. The smaller values of GB conductivity manifest themselves in a lower overall thermal conductivity, but the main trend holds, and a grain size of $100 \mathrm{~nm}$ still appears to be the crossover where thermal transport is no longer dominated by the GBs.

To summarize, we have presented an electrical and thermal characterization of coplanar G-hBN heterostructures. The tight-binding model includes a refined description of the $\mathrm{G}$ $\mathrm{hBN}$ interfaces, and is used to describe the electrical properties of polycrystalline structures with varying percentages of graphene and $\mathrm{hBN}$. Our results reproduce the transition from graphene to insulating $\mathrm{hBN}$, with an electrical conductivity change of more than two orders of magnitude and a strong suppression of the Seebeck coefficient. Additionally, the thermal conductivity of these polycrystalline structures has been investigated using a combination of atomistic MD simulations and a FE evaluation of the heat equation. We find that for small-grain structures, the thermal conductivity is minimized for a hBN grain density of $70 \%$. From our study, we can evaluate the upper value of the thermoelectric figure of merit, $Z T=\sigma S^{2} T / \kappa$. For example, in the case of $40 \mathrm{~nm}$ average grain size and $20 \% \mathrm{hBN}, Z T \sim 1 \times 10^{-4}$ for a carrier concentration $n=5 \times 10^{12} \mathrm{~cm}^{-2}$, which is quite small. Even for energies near the edge of the gap, where the Seebeck coefficient should be maximized, the value of $Z T$ only reaches $\sim 1 \times 10^{-2}$.

Acknowledgement J.E.B.-V. acknowledges support from CONACyT (Mexico, D.F.). This work was supported by European Union Seventh Framework Programme under grant agreement 604391 Graphene Flagship (R.M.G.). S. R. acknowledges the Spanish Ministry of Economy and Competitiveness for funding (MAT2012-33911), the Secretaria de Universidades e Investigacion del Departamento de Economia y Conocimiento de la Generalidad de Cataluna and the Severo Ochoa Program (MINECO SEV-2013-0295). M.P. and L.C. acknowledge Spanish MINECO (FIS201564886-C5-3-P) and Generalitat de Catalunya (2014SGR301). B.M. and T.R. greatly acknowledge the financial support by European Research Council for COMBAT project (Grant number 615132).

Supporting Information Available: Details of tight-binding model, details on the numerical evaluation of electrical and thermal conductivity, and scaling analysis to estimate electrical and thermal GB resistivity. This material is available free of charge via the Internet at http://pubs.acs . org/. 


\section{References}

(1) Rubio, A. Nat. Mater. 2010, 9, 379.

(2) Ci, L.; Song, L.; Jin, C.; Jariwala, D. et al. Nat. Mater. 2010, 9, 430.

(3) Novoselov, K. S.; Geim, A. K.; Morozov, S. V.; Jiang, D. et al. Science 2004, 306, 666-669.

(4) Balandin, A. A.; Ghosh, S.; Bao, W.; Calizo, I. et al. Nano Lett. 2008, 8, 902-907.

(5) Jo, I.; Pettes, M. T.; Kim, J.; Watanabe, K. et al. Nano Lett. 2013, 13, 550554 .

(6) Wang, C.; Guo, J.; Dong, L.; Aiyiti, A. et al. Sci. Rep. 2016, 6, 25334.

(7) Levendorf, M. P.; Kim, C.-J.; Brown, L.; Huang, P. Y. et al. Nature 2012, 488, 627632 .

(8) Liu, Z.; Ma, L.; Shi, G.; Zhou, W. et al. Nat. Nanotechnol. 2013, 8, 119-124.

(9) Han, G. H.; Rodríguez-Manzo, J. A.; Lee, C.-W.; Kybert, N. J. et al. ACS Nano 2013, 7, 10129-10138.

(10) Liu, L.; Park, J.; Siegel, D. A.; McCarty, K. F. et al. Science 2014, 343, 163167.

(11) Zhao, R.; Wang, J.; Yang, M.; Liu, Z. et al. The Journal of Physical Chemistry C 2012, 116, 21098-21103.

(12) Matthes, L.; Hannewald, K.; Bechstedt, F. Phys. Rev. B 2012, 86, 205409.

(13) Gong, Y.; Shi, G.; Zhang, Z.; Zhou, W. et al. Nat. Commun. 2014, 5 .

(14) Song, L.; Balicas, L.; Mowbray, D. J.; Capaz, R. B. et al. Phys. Rev. B 2012, 86, 075429 .

(15) Tuan, D. V.; Kotakoski, J.; Louvet, T.; Ortmann, F. et al. Nano Lett. 2013, 13, 1730-1735.
(16) Cummings, A. W.; Duong, D. L.; Nguyen, V. L.; Van Tuan, D. et al. Adv. Mater. 2014, 26, 5079-5094.

(17) Mortazavi, B.; Potschke, M.; Cuniberti, G. Nanoscale 2014, 6, 3344-3352.

(18) Hahn, K. R.; Melis, C.; Colombo, L. Carbon 2016, 96, 429-438.

(19) Isacsson, A.; Cummings, A. W.; Colombo, L.; Colombo, L. et al. 2D Materials 2017, 4, 012002.

(20) Ma, C.; Sun, H.; Zhao, Y.; Li, B. et al. Phys. Rev. Lett. 2014, 112, 226802.

(21) Tison, Y.; Lagoute, J.; Repain, V.; Chacon, C. et al. Nano Lett. 2014, 14, 63826386 .

(22) Luican-Mayer, A.; Barrios-Vargas, J. E.; Falkenberg, J. T.; Autès, G. et al. 2D Mater. 2016, 3, 031005.

(23) Li, Q.; Zou, X.; Liu, M.; Sun, J. et al. Nano Lett. 2015, 15, 5804-5810.

(24) Drost, R.; Uppstu, A.; Schulz, F.; Hämäläinen, S. K. et al. Nano Lett. 2014, $14,5128-5132$.

(25) Lu, J.; Gomes, L. C.; Nunes, R. W.; Neto, A. H. C. et al. Nano Lett. 2014, 14, 5133-5139.

(26) Wang, Y.; Song, Z.; Xu, Z. J. Mater. Res. 2014, 29, 362-372.

(27) Liu, H. K.; Lin, Y.; Luo, S. N. J. Phys. Chem. C 2014, 118, 24797-24802.

(28) Mortazavi, B.; Pereira, L. F. C.; Jiang, J.W.; Rabczuk, T. Sci. Rep. 2015, 5.

(29) Plimpton, S. J. Comp. Phys. 1995, 117, $1-19$.

(30) Brenner, D. W.; Shenderova, O. A.; Harrison, J. A.; Stuart, S. J. et al. J. Phys.: Condens. Matter 2002, 14, 783. 
(31) Martínez-Gordillo, R. Atomistic simulations in hybrid $\mathrm{C} / \mathrm{BN}$ structures. Ph.D. thesis, Universitat Autònoma de Barcelona, 2014; Chapter 5.

(32) Roche, S. Phys. Rev. B 1999, 59, 22842291.

(33) Torres, L. E. F. F.; Roche, S.; Charlier, J.C. Introduction to Graphene-Based Nanomaterials; Cambridge University Press: Cambridge, UK, 2014.

(34) Dorgan, V. E.; Bae, M.-H.; Pop, E. Appl. Phys. Lett. 2010, 97.

(35) Woessner, A.; Alonso-González, P.; Lundeberg, M. B.; Gao, Y. et al. Nat. Commun. 2016, 7, 10783.

(36) Sevinçli, H.; Li, W.; Mingo, N.; Cuniberti, G. et al. Phys. Rev. B 2011, 84, 205444 .

(37) Kınacı, A.; Haskins, J. B.; Sevik, C.; Çağın, T. Phys. Rev. B 2012, 86, 115410.

(38) Melis, C.; Dettori, R.; Vandermeulen, S.; Colombo, L. Eur. Phys. J. B 2014, 87, 96. 


\section{Supporting Information:}

\section{Electrical and thermal transport in coplanar polycrystalline graphene-hBN heterostructures}

J.E. Barrios Vargas, ${ }^{* \dagger}$ B. Mortazavi, ${ }^{\ddagger}$ A.W. Cummings, ${ }^{\dagger}$ R. Martinez-Gordillo, M. Pruneda, ${ }^{\dagger}$ L. Colombo, ${ }^{\dagger, \S}$ T. Rabczuk, ${ }^{\ddagger}$ and S. Roche ${ }^{*, \dagger, \|}$

$\dagger$ Catalan Institute of Nanoscience and Nanotechnology (ICN2), CSIC and The Barcelona Institute of Science and Technology, Campus UAB, 08193 Barcelona, Spain $\ddagger$ Institute of Structural Mechanics, Bauhaus-Universität Weimar, Marienstr. 15, D-99423

Weimar, Germany

\CINaM - Centre Interdisciplinaire de Nanoscience de Marseille, France

$\S$ Dipartimento di Fisica, Universit di Cagliari, Cittadella Universitaria, I-09042

Monserrato (Ca), Italy

||ICREA - Institució Catalana de Recerca i Estudis Avançats, 08010 Barcelona, Spain

E-mail: jose.barrios@icn2.cat; stephan.roche@icn2.cat

\section{Tight-Binding Model}

In systems containing 1D interfaces between two different 2D materials, the electronic properties are sensitive to interface termination. Zigzag edges of both graphene and hBN should be treated carefully when modeling in a phenomenological way. On the one hand, zigzag graphene nanoribbons (ZGNRs) can become half-metals by the effect of a transverse electric field. ${ }^{1}$ On the other hand, zigzag hBN NRs are polar, presenting bound charge of opposite 
signs at the $\mathrm{B}$ and $\mathrm{N}$ edges.

For zigzag interfaces, the gap of the hybrid system will depend on the widths of both the graphene and the hBN, becoming smaller for wider ribbons (with a more important contribution from graphene for the closing of the gap, since its gap decays to zero more quickly than in ZBNNRs). Since ZBNNRs and polar and ZGNRs nonpolar, there is a polar discontinuity at the interface, which gives an excess interfacial bound charge that can be calculated by the interface theorem formulated by Vanderbilt and King-Smith, ${ }^{2,3}$ which states that

$$
\left(\mathbf{P}_{1}-\mathbf{P}_{2}\right) \cdot \hat{\mathbf{n}}=\sigma_{\mathrm{b}}
$$

As graphene has inversion symmetry, the bulk polarization is taken as zero. Thus, the bound charge at the interface is $\sigma_{\text {int }}= \pm e a_{0}$, being positive at the $\mathrm{C}$-B interface and negative at the C-N edge. This form of determining the interface charge of coplanar hybrids of polar compounds with hexagonal lattices has been used previously, showing that at the zigzag edges there forms a one-dimensional electron/hole gas. ${ }^{4}$ Mobile electrons from the ZGNR will tend to screen this excess charge at the interface by transferring electrons from one edge to the other, which will change the potential profile across the ribbon and produce an effective electric field capable of inducing half-metallicity when it is strong enough to produce a Zener-like breakdown. For wider ZGNRs, the gap is so small for both spins that it is very easy to close in the hybrid systems, which then become metallic. ${ }^{5}$

To model the effect of polarization produced by edge effects in BNNRs, the on-site term is modified to include an electrostatic potential. In this way, the new position-dependent on-site energy becomes

$$
\varepsilon_{\alpha}(\mathbf{r})=\varepsilon_{\alpha 0}-e \Phi(\mathbf{r})
$$

where $\Phi$ is an effective potential ${ }^{6}$ that is considered to be a decaying function that vanishes 
in the centre of the ribbon.

The parameters for the tight-binding (TB) model are computed from a Hamiltonian expressed in the basis of Wannier functions (WF), which are obtained via wannierization of the Bloch states calculated from first principles. The density functional theory (DFT) calculations are done using norm-conserving Troullier-Martins pseudopotentials. ${ }^{7}$ and the PBE generalized gradient approximation ${ }^{8}$ as implemented in the SIESTA code. ${ }^{9}$ The wavefunctions for the valence electrons are described using a linear combination of pseudo-atomic numerical orbitals with a double- $\zeta$ polarized (DZP) basis. ${ }^{10} \mathrm{~A}$ vacuum space of $\sim 40 \AA$ is taken to prevent interactions between between layers under periodic boundary conditions. The atomic positions are relaxed until the forces are smaller than $0.01 \mathrm{eV} / \AA$. Once the density matrix is converged the expansion coefficients of the Bloch wave functions obtained from the SIESTA Kohn-Sham Hamiltonian, $c_{\mu n}$, can be used to obtain the unitary matrix $U_{m n}(\mathbf{k})$ necessary for the construction of the WF. ${ }^{11}$

For both graphene and hBN, a good choice for the trial functions $g_{n}(\mathbf{r})$ is to represent the electronic orbitals as $\mathrm{sp}^{2}$ and $\mathrm{p}_{\mathrm{z}}$. Then, for each unit cell with two atoms, there are three $\mathrm{sp}^{2}$ and two $\mathrm{p}_{\mathrm{z}}$ orbitals. This gives a total of five basis functions, of which four are occupied states and one is an empty state, enough to achieve a good description of the valence bands and the first conduction band. Since the first conduction band crosses higher energy bands, a band disentanglement procedure is used $^{12}$ following the prescription implemented in the WANNIER90 code $^{13}$ for maximally localised WF (MLWF). The Hamiltonian in the basis of MLWF can be seen as a representation of the interactions between localized functions that, by construction of the trial orbitals, have great resemblance with the chemical $\sigma$-and $\pi$-bonds of graphene and hBN. Consequently, the relevant TB parameters can be straightforwardly extracted from this Hamiltonian.

The systems considered to make the parametrization were constructed by joining graphene and BN nanoribbons so that heterojunctions with zigzag or armchair interfaces were obtained. A diagram of the systems is shown in Figure S1(a). The number of C zigzag or 
armchair chains in the system is labeled $n_{\mathrm{C}}$, while $n_{\mathrm{BN}}$ designates those for hBN. Systems with compositions in the range of $5 \leq n_{\mathrm{C}} \leq 9$ and $5 \leq n_{\mathrm{BN}} \leq 12$ were studied in the case of zigzag interfaces. For armchair systems, values of $9 \leq n_{\mathrm{C}} \leq 12$ and $12 \leq n_{\mathrm{BN}} \leq 15$ were used.
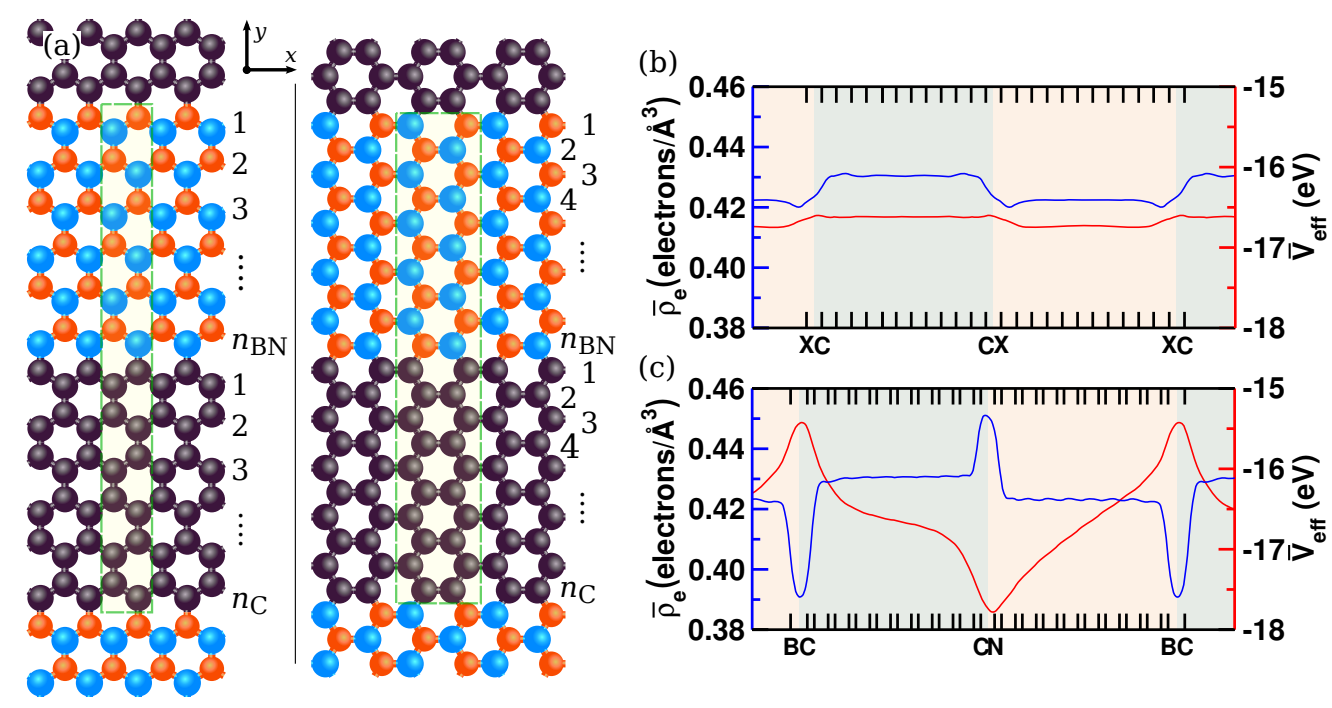

Figure S1: (a) Diagram of the hybrid systems consisting of nanoribbons of graphene and hBN joined by the zigzag (left) and armchair (right) interface. The systems are defined by the number of rows of each material, denoted $n_{\mathrm{C}}$ for graphene and $n_{\mathrm{BN}}$ for hBN. The unit cell is enclosed in the dashed green box. (b) Macroscopic average of the electronic charge density (blue) and effective potential (red) across the $y$ direction for the armchair system with $n_{\mathrm{C}}=n_{\mathrm{BN}}=12$. (c) The same as (b) for the zigzag system with $n_{\mathrm{C}}=n_{\mathrm{BN}}=9$.

The macroscopic average of the electronic charge density ${ }^{14}$ and the effective potential are displayed for an armchair and a zigzag system, in Figure S1(b) and Figure S1(c), respectively. At the interface of armchair ribbons, there is a soft change in the charge density due to charge redistribution at the $\mathrm{C}-\mathrm{B}$ and $\mathrm{C}-\mathrm{N}$ bonds. In the zigzag case, abrupt changes in the charge density can be observed at the interface. The accumulation of electrons in the $\mathrm{C}-\mathrm{N}$ side and a depletion in the C-B interface comes from the bound charges of the polarization discontinuity. To counteract the effect of hBN polarization, free carriers from graphene must screen out the bound charges by moving electrons from the $\mathrm{C}-\mathrm{N}$ side to the $\mathrm{C}-\mathrm{B}$ side. The result is a lack of electrons in the net charge at the $\mathrm{C}-\mathrm{N}$ interface and an excess of electron in the net charge at the $\mathrm{C}-\mathrm{B}$ interface. The localized charges at the interface cause a change in 
the potential as seen in Figure S1(c), and is essential to model the electronic properties of hybrid systems with zigzag interface. The potential has a maximum at the $\mathrm{C}-\mathrm{B}$ interface, with less charge, and a minimum at the $\mathrm{C}-\mathrm{N}$ interface, with more charge.

The charges at the 1D interfaces can be simplistically seen as two lines of charge, placed at $\pm L / 2$ and having a linear charge density $\pm Q_{\lambda}$. This is shown schematically in Figure S2(a). The problem of a line of charge is well known in electrostatics, ${ }^{15}$ that in this case with two oppositely-charged lines results in the potential

$$
\Phi(x)=\frac{Q_{\lambda}}{2 \pi \epsilon_{0}} \ln \left(\frac{L+2 x}{L-2 x}\right),
$$

where $\epsilon_{0}$ is the vacuum permittivity. This potential is plotted in Figure S2(a), and has a similar shape to the one shown in Figure S1(c). The differences in the functional shape come from the fact that the potential from the DFT calculation corresponds to a periodically repeated array of linear charges, which have some spatial spread (more similar to a cylindrical charge distribution) and act over different materials, each one with its own permittivity, which leads to a different screening due to valence electrons in each region. For the model to be computationally efficient, instead of considering an infinite line it is better to discretize the problem considering an infinite array of point charges along the interfacial axis, as shown in Figure S2(b). The position of the point charges is taken to be midway between the C-B and $\mathrm{C}-\mathrm{N}$ interfacial bonds.

Due to the semi-metallicity of graphene, the electric field in this region will be damped by the redistribution of valence electrons. The screened Poisson equation describes systems with this kind of screened Coulomb interaction, and here is considered to model the interfacial potential. The screened potential for an arrange of point charges has the form

$$
\Phi(r)=\sum_{i} \frac{Q_{i}}{4 \pi \epsilon_{0} r_{i}} e^{-\frac{r_{i}}{\lambda}}
$$

where $Q_{i}$ are the charges, $r_{i}$ is the distance between $r$ and the charge $Q_{i}$, and $\lambda$ is a damping 


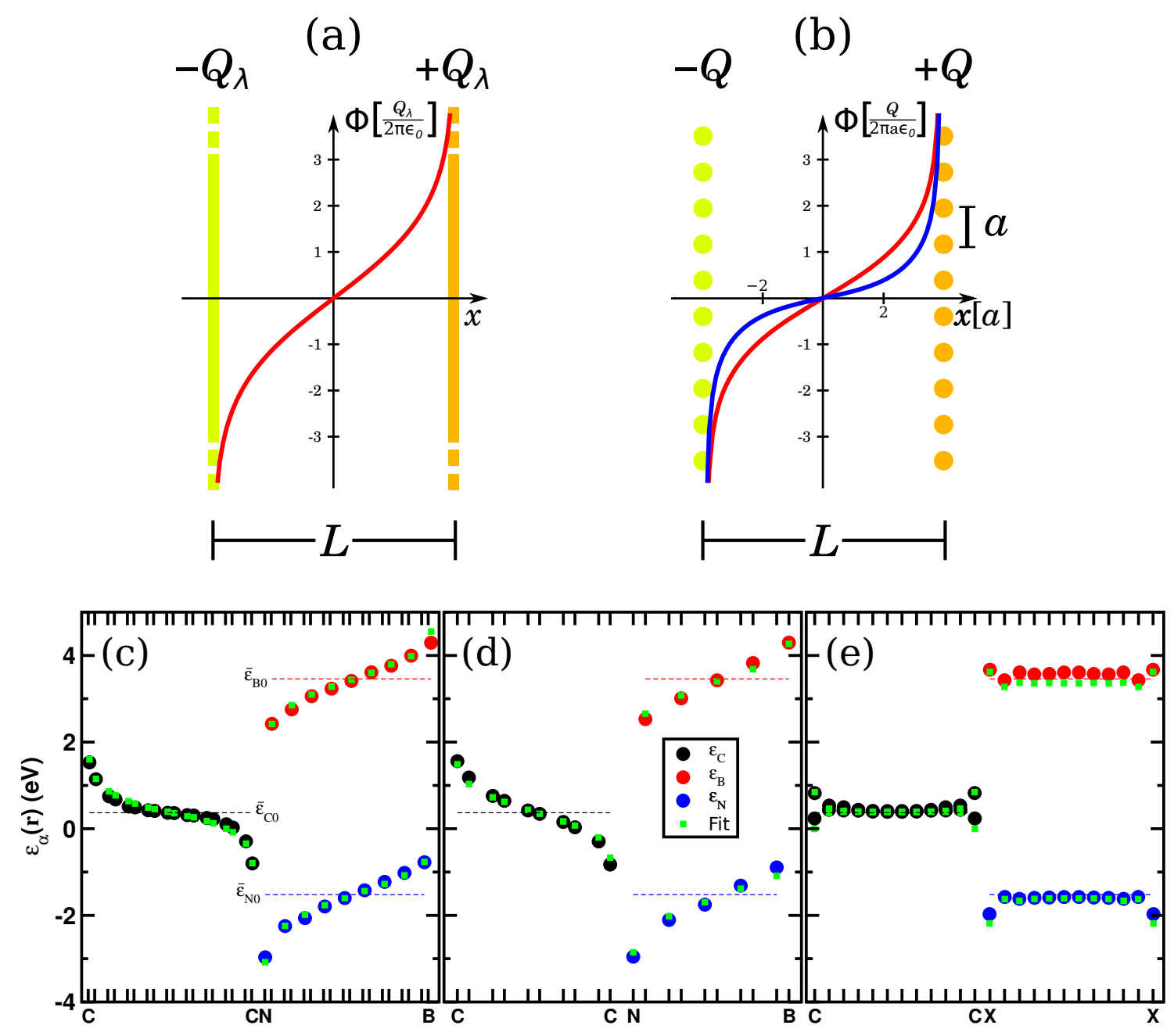

Figure S2: (a) Parallel charged lines with opposite charges $\left( \pm Q_{\lambda}\right)$ situated at $\pm L / 2$. (b) Discretized problem with point charges $\pm Q$ situated at $\pm L / 2$. At each side, the charges are equally spaced at a distance $a$. The value $L=8 a$ is considered. Two different damping factors $(\lambda)$ of the screened potential are used, $\lambda=7 a$ (red) and $\lambda=2 a$ (blue). (c-e) On-site energy values as a function of the atomic positions for the systems (c) $Z_{9,9}$, (d) $Z_{5,5}$ and (e) $\mathrm{A}_{12,12}$. Black circles correspond to $\mathrm{C}$ atoms, red circles to $\mathrm{B}$ and blue circles to N. Green squares are the on-site terms $\varepsilon_{\alpha}(\mathbf{r})$ with the functional form in Eq. (5) and the parameters from Table S1. 
factor related to the mobility of electrons in the material. The potential profile in the graphene part in Figure S1(b) looks more like the screened potential with short $\lambda$, since the availability of electrons to screen the potential is large. The effect of the potential can be included in the on-site term of the TB Hamiltonian by considering the sum of two screenedlike potentials with opposite signs, corresponding to both interfaces. This gives

$$
\varepsilon_{\alpha}(\mathbf{r})=\bar{\varepsilon}_{\alpha 0}+\sum_{i}^{n_{q}} \frac{A_{\alpha}^{\mathrm{B}}}{\left|\mathbf{r}-\mathbf{r}_{i}^{\mathrm{B}}\right|} e^{-\frac{\left|\mathbf{r}-\mathbf{r}_{i}^{\mathrm{B}}\right|}{\lambda_{\alpha}}}-\sum_{i}^{n_{q}} \frac{A_{\alpha}^{\mathrm{N}}}{\left|\mathbf{r}-\mathbf{r}_{i}^{\mathrm{N}}\right|} e^{-\frac{\left|\mathbf{r}-\mathbf{r}_{i}^{\mathrm{N}}\right|}{\lambda_{\alpha}}},
$$

which represents the diagonal term for an atom of species $\alpha$ at position $\mathbf{r}$, where $\bar{\varepsilon}_{\alpha 0}$ is the on-site energy in absence of the potential, $A_{\alpha}^{\mathrm{B}}$ and $A_{\alpha}^{\mathrm{N}}$ are the amplitudes of the potential for that species in the $\mathrm{B}$ and $\mathrm{N}$ interfaces, respectively, $\mathbf{r}_{i}^{\mathrm{B}}$ and $\mathbf{r}_{i}^{\mathrm{N}}$ are the positions of the charges at the $\mathrm{C}-\mathrm{B}$ and $\mathrm{C}-\mathrm{N}$ interfaces, respectively, $\lambda_{\alpha}$ is the damping factor, and the sum is done for all $n_{q}$ charges in the unit cell and in all periodically repeated neighbour cells within the cutoff radius $r_{\text {cut }}$. The parameter $r_{\text {cut }}$ is taken so that changes in the on-site term due to point charges far away are less than $0.02 \mathrm{eV}$, which gives $r_{\text {cut }} \sim 10 \AA$. The amplitudes of the potential for $\mathrm{B}$ and $\mathrm{N}$ are taken to be equal $\left(A_{\mathrm{B}}^{\mathrm{B}}=A_{\mathrm{N}}^{\mathrm{B}}\right.$ and $\left.A_{\mathrm{B}}^{\mathrm{N}}=A_{\mathrm{N}}^{\mathrm{N}}\right)$, and so are their damping lengths $\left(\lambda_{\mathrm{B}}=\lambda_{\mathrm{N}}\right)$.

The parameters $A_{\alpha}^{\mathrm{B}}, A_{\alpha}^{\mathrm{N}}, \lambda_{\alpha}$ and $\bar{\varepsilon}_{\alpha 0}$ are fitted to the data from the diagonal of the Wannier Hamiltonian using the simplex algorithm. ${ }^{16}$ As shown in Figure S2(c-e), the on-site energies from this Hamiltonian are in good agreement with the form of the potential in S5, and the fitted values reproduce well these on-site energies. The obtained parameters for the on-site potential are shown in Table S1. Although the on-site energies are fitted from zigzag

Table S1: Parameters obtained from the fit to the on-site energies of the zigzag systems.

\begin{tabular}{|c|c|c|c|}
\hline & $\mathrm{C}$ & B & $\mathrm{N}$ \\
\hline $\bar{\varepsilon}_{\alpha 0}(\mathrm{eV})$ & 0.00 & 3.09 & -1.89 \\
\hline$A_{\alpha}^{\mathrm{B}}(\mathrm{eV} \cdot \hat{A})$ & 0.61 & 0.46 & 0.46 \\
\hline$A_{\alpha}^{\mathrm{N}}(\mathrm{eV} \cdot \AA)$ & 0.54 & 0.62 & 0.62 \\
\hline$\lambda_{\alpha}(\AA)$ & 6.78 & 12.56 & 1256 \\
\hline
\end{tabular}


systems, they also give a good description of armchair systems.

\section{Electrical properties}

The local density of states of the grain boundary $\left(\operatorname{LDOS}_{\mathrm{GB}}\right)$ shows several main peaks as the concentration of $\mathrm{hBN}$ is increased (Figure 2(b) in the main text), these peaks corresponds to $E=-1.2,-0.5,0.0,0.76$ and $2.05 \mathrm{eV}$. In order to understand the origin of the peaks we plot the $\mathrm{LDOS}_{\mathrm{GB}}$ projected around a G-hBN interface (Figure S3). The peaks at $E=-1.20$ and $2.05 \mathrm{eV}$ are close to the energies experimentally observed for grain boundaries in $\mathrm{hBN}^{17}$. Furthermore, the peaks at $E=0.0$ and $0.76 \mathrm{eV}$ are found for graphene and hBN, which suggest specific fingerprints of the structural morphology of grain boundaries.

In the real-space order- $\mathrm{N}$ wave packet propagation method ${ }^{18,19}$ we evaluate the timedependent diffusion coefficient using the mean-square displacement of the wave packet, $\Delta X^{2}$,

$$
D(E, t)=\frac{\partial}{\partial t} \Delta X^{2}(E, t)
$$

where

$$
\Delta X^{2}(E, t)=\frac{\operatorname{Tr}\left[\delta(E-\hat{H})|\hat{X}(t)-\hat{X}(0)|^{2}\right]}{\rho(E)},
$$

and $\rho(E)=\operatorname{Tr}[\delta(E-\hat{H})]$ is the density of states (DOS). Ambiguity in the time dependency can be avoided by noting the convergence of the diffusion coefficient as the wave packet is spreading. This convergence depends on the mean displacement of the wavepacket, $\sqrt{\Delta X^{2}}$, which is energy dependent. We use six times the average grain size $\left(6 L_{\text {grain }}\right)$ as the point of convergence, and at that particular mean displacement we extract the diffusion coefficient $\tilde{D}(E)$ and we evaluate the semiclassical conductivity, $\sigma(E)=e^{2} \rho(E) \tilde{D}(E)$. As an example, we show in Figure S4 the conductivity as a function of the mean displacement. It is worth 

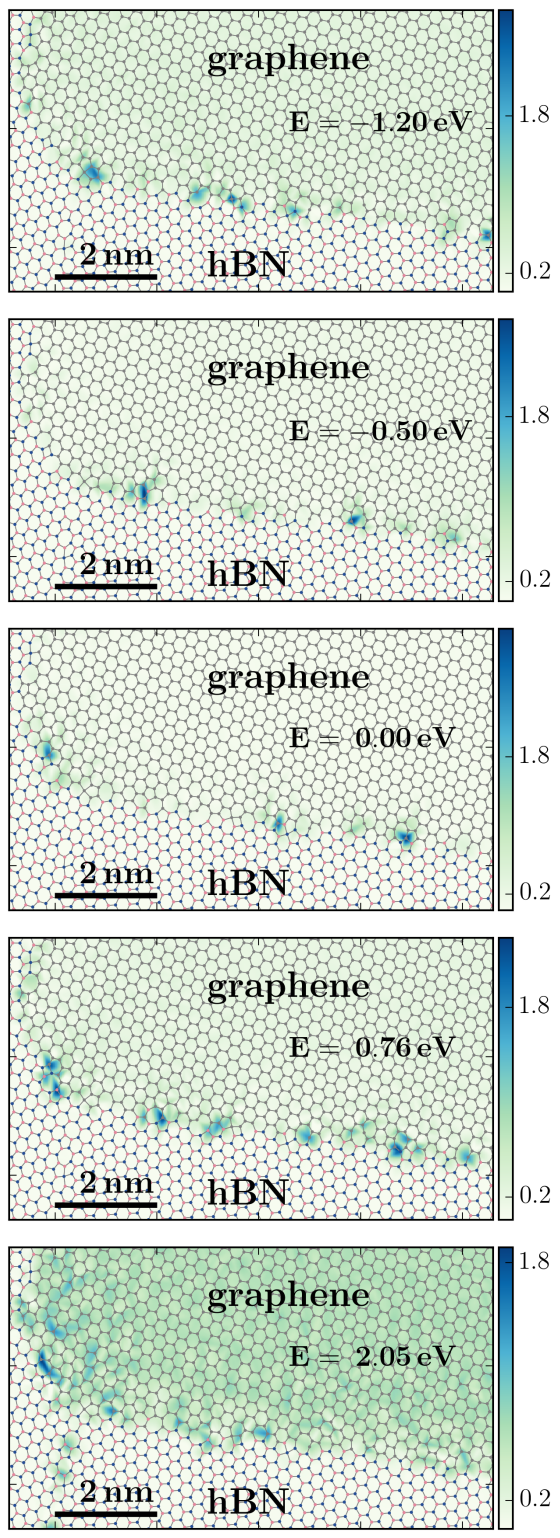

Figure S3: LDOS $_{\mathrm{GB}}$ projected around a G-hBN interface, corresponding to a fix energy labeled in each frame. 
mentioning that the time to reach $6 L_{\text {grain }}$ is highly dependent on the concentration of $\mathrm{hBN}$; a lower conductivity corresponds to a longer time to reach a particular mean displacement.

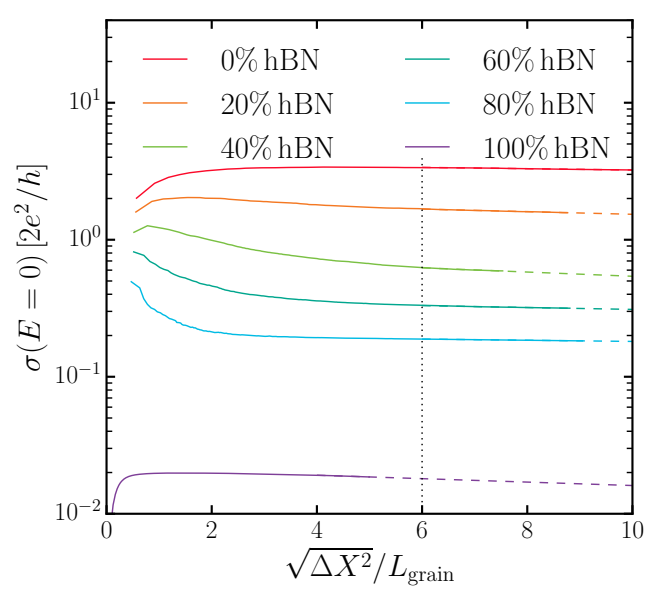

Figure S4: Electrical conductivity $(\sigma)$ as a function of normalized mean spreading $\sqrt{\Delta X^{2}} / L_{\text {grain }}$ for $E=0$.

In order to extract the GB resistivity, $\rho_{\mathrm{GB}}$, we evaluate the electrical conductivity in polycrystalline samples with three different average grain sizes $\left(L_{\text {grain }}=10,20\right.$ and $\left.40 \mathrm{~nm}\right)$ for different concentrations of $\mathrm{hBN}(0,20,40,60,80$ and 100\%). The polycrystalline samples are square, so the sheet resistance $R=(1 / \sigma) \times L / W=1 / \sigma$. We use the ohmic scaling analysis of Cummings et al. ${ }^{20}$ in order to fit $\rho_{\mathrm{GB}}$ (Figure S5),

$$
R=R^{0}+\frac{\rho_{\mathrm{GB}}}{L_{\text {grain }}}
$$

where $R$ and $R^{0}$ are the sheet resistances of the polycrystalline sample and the individual grains, respectively. Applying the scaling analysis to the $0 \%$ and $100 \%$ cases, we find the GB resistivity and sheet resistance of graphene to be $0.12 \mathrm{k} \Omega \cdot \mu \mathrm{m}$ and $1.1 \mathrm{k} \Omega$, respectively. The values for $\mathrm{hBN}$ are $5.93 \mathrm{k} \Omega \cdot \mu \mathrm{m}$ and $608.87 \mathrm{k} \Omega$. Owing to the large bandgap in the $\mathrm{hBN}$ grains, charge transport through the polycrystalline hBN sample will be primarily through the disordered network of GBs, explaining the large values of sheet resistance and GB resistivity. 


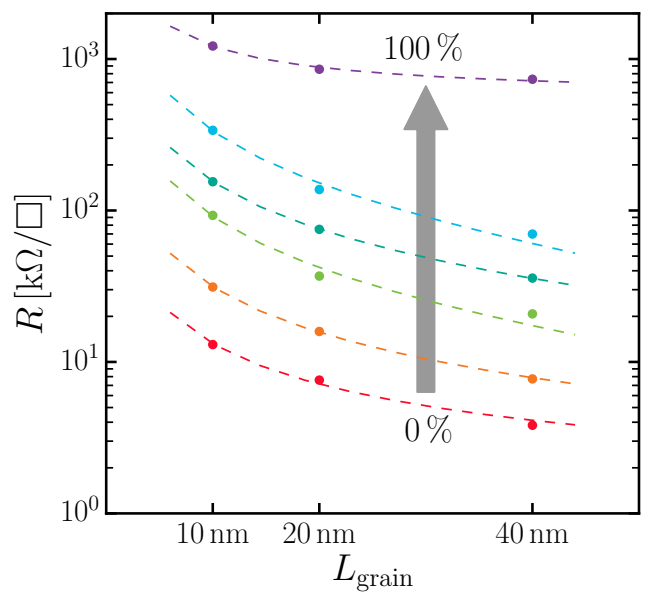

Figure S5: Sheet resistance as a function of the grain size $L_{\text {grain }}$, for different values of hBN grain concentration.

\section{Thermal properties}

\section{Thermal conductance of individual GBs}

The repesentative GB conductance is an arithmetic average of the six type of GBs shown in Figure S6. These $\mathrm{GBs}$ are constructed for $\mathrm{G}-\mathrm{G}, \mathrm{hBN}-\mathrm{hBN}$ and $\mathrm{G}-\mathrm{hBN}$ interfaces and are periodic along the GB direction. We evaluate the GB conductance for each GB using a nonequilibrium molecular dynamics (NEMD) calculation using the Tersoff potential developed for boron, carbon and nitrogen interactions. ${ }^{21}$ To perform a NEMD calculation, we first fix the borders and divide the simulation box (excluding the fixed atoms) along the length into 22 slabs. Second, we thermalize the structure using NVT at room temperature, and then we apply a $20 \mathrm{~K}$ temperature difference between the first (hot slab) and the 22nd slabs (cold slab). Third, we let the system evolve using an NVE ensemble and we keep the borders, hot and cold, at the desired temperature difference using an NVT thermostat. Fourth, we 
evaluate the heat-flux using,

$$
q_{x}=\frac{1}{A} \frac{d q}{d t},
$$

where $A$ is the cross sectional area of the sheet and $q$ is the energy added or removed from the system. The steady state process is achieved after around $500 \mathrm{ps}$ of simulation time. Finally, we evaluate the thermal conductivity $\kappa_{\mathrm{s}}$ using the one-dimensional Fourier law,

$$
\kappa_{\mathrm{s}}=q_{x} \frac{d T}{d x} .
$$

The temperature profile is linear along the sample except in the middle where there is a jump, $\Delta T$, due to the GB resistance. Therefore, the GB conductance, $C_{\mathrm{GB}}$, is evaluated as

$$
C_{\mathrm{GB}}=\frac{q_{x}}{\Delta T} .
$$

In Table S2, the conductances of the $\mathrm{G}-\mathrm{G}, \mathrm{hBN}-\mathrm{hBN}$ and $\mathrm{G}-\mathrm{hBN}$ GBs at room temperature are presented.

Table S2: Calculated GB conductance for different grain boundary types (as shown in Figure $\mathrm{S} 6$ ) and for $\mathrm{G}-\mathrm{G}, \mathrm{hBN}-\mathrm{hBN}$ and $\mathrm{G}-\mathrm{hBN}$ interfaces. The results are for a temperature of $300 \mathrm{~K}$.

\begin{tabular}{c|ccc} 
GB type & $C_{\mathrm{G}-\mathrm{G}}\left[\mathrm{GW} / \mathrm{m}^{2} \mathrm{~K}\right]$ & $C_{\mathrm{hBN}-\mathrm{hBN}}\left[\mathrm{GW} / \mathrm{m}^{2} \mathrm{~K}\right]$ & $C_{\mathrm{G}-\mathrm{hBN}}\left[\mathrm{GW} / \mathrm{m}^{2} \mathrm{~K}\right]$ \\
\hline 1 (symmetric) & $36.5 \pm 3$ & $14.5 \pm 1.5$ & $8.5 \pm 1.5$ \\
2 (symmetric) & $49 \pm 3$ & $19 \pm 1.5$ & $10 \pm 1.5$ \\
3 (non-symmetric) & $48 \pm 3$ & $18.5 \pm 1.5$ & $9 \pm 1.5$ \\
4 (symmetric) & $68 \pm 4$ & $25.5 \pm 2$ & $11 \pm 1.5$ \\
5 (non-symmetric) & $48 \pm 3$ & $21 \pm 1.5$ & $10 \pm 1.5$ \\
6 (symmetric) & $69 \pm 4$ & $29.5 \pm 2$ & $11.5 \pm 1.5$ \\
Defect-free & - & - & $15 \pm 1.5$ \\
\hline
\end{tabular}

In order to extract the thermal conductance from approach to equilibrium molecular dynamics (AEMD) calculations, we fit the thermal conductivity as a function of the average 


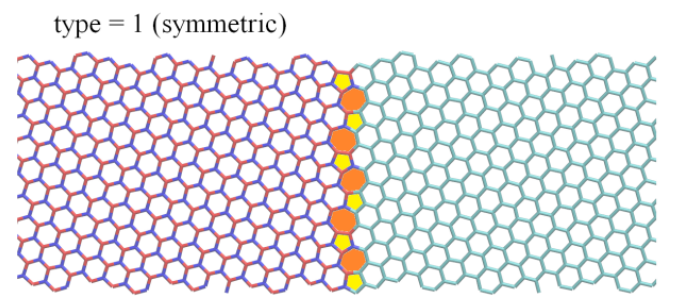

type $=3$ (non-symmetric)

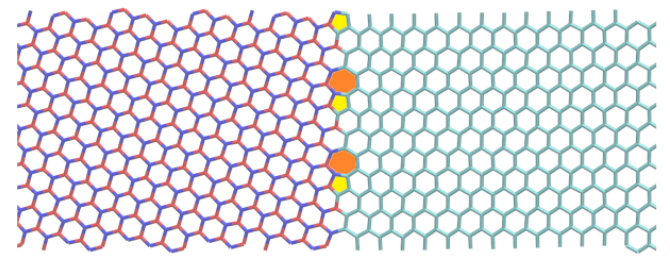

type $=5$ (non-symmetric)

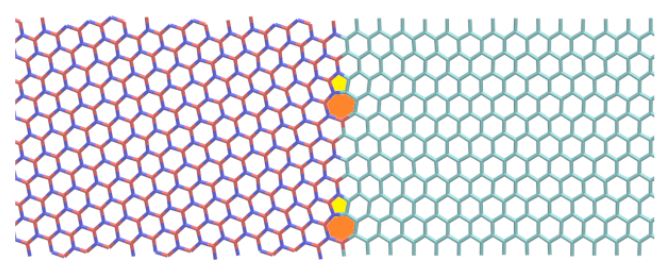

type $=2($ symmetric $)$

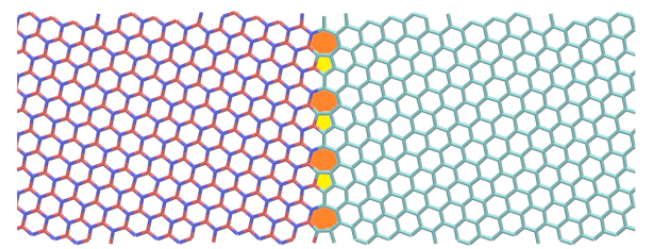

type $=4$ (symmetric)

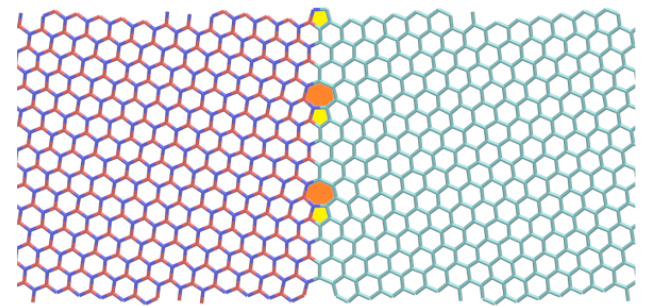

type $=6$ (symmetric)

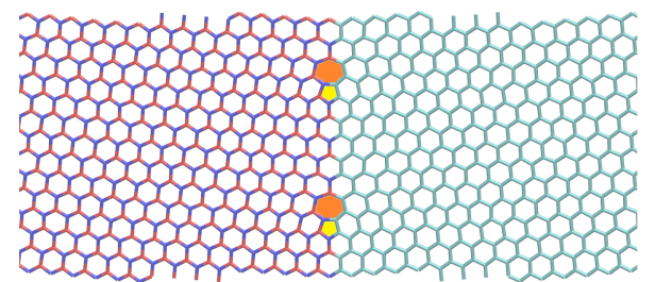

Figure S6: Atomic structure of 6 different grain boundaries consisting of pentagon/heptagon pairs with various concentrations. These models were constructed for $\mathrm{G}-\mathrm{G}, \mathrm{hBN}-\mathrm{h}-\mathrm{BN}$ and $\mathrm{G}-\mathrm{hBN}$ grain boundaries. 
grain size using, ${ }^{22}$

$$
\frac{1}{\kappa}=\frac{1}{\kappa_{\mathrm{c}-\mathrm{hBN}}}+\frac{R_{\mathrm{GB}}}{2 L_{\text {grain }}}
$$

where $\kappa_{\mathrm{c}-\mathrm{hBN}}$ is the thermal conductivity of the pristine $\mathrm{hBN}$ and $C_{\mathrm{hBN}-\mathrm{hBN}}=1 / R_{\mathrm{GB}}$ is the thermal conductance. In Figure S7 we show the fit for hBN-hBN interface. For graphene we extract the values from Ref. ${ }^{22}$, which are also shown in Figure S7.

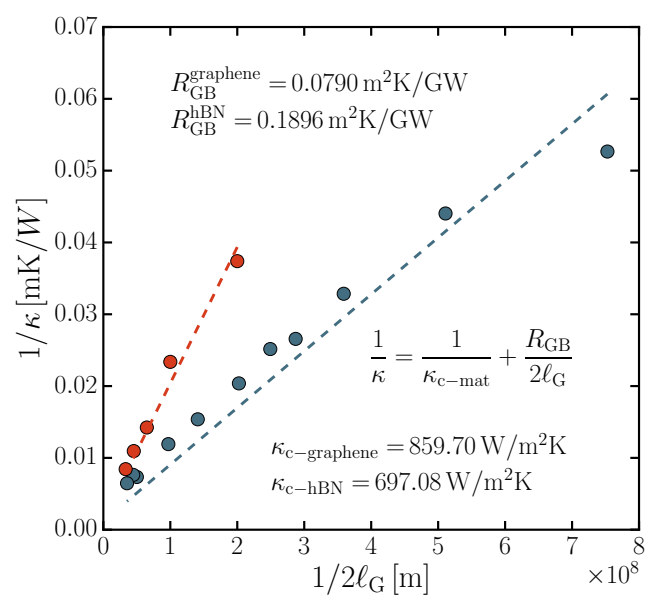

Figure S7: Thermal resistance estimation using AEMD calculations. The graphene calculations are values extracted from Ref. ${ }^{22}$ and the hBN values are results from this work.

\section{References}

(1) Son, Y.-W.; Cohen, M. L.; Louie, S. G. Nature 2006, 444, 347-349.

(2) Vanderbilt, D.; King-Smith, R. D. Phys. Rev. B 1993, 48, 4442-4455.

(3) Stengel, M.; Vanderbilt, D. Phys. Rev. B 2009, 80, 241103.

(4) Bristowe, N. C.; Stengel, M.; Littlewood, P. B.; Artacho, E.; Pruneda, J. M. Phys. Rev. $B$ 2013, 88, 161411.

(5) Pruneda, J. M. Phys. Rev. B 2010, 81, 161409. 
(6) Graf, M.; Vogl, P. Phys. Rev. B 1995, 51, 4940-4949.

(7) Troullier, N.; Martins, J. L. Phys. Rev. B 1991, 43, 1993-2006.

(8) Perdew, J. P.; Burke, K.; Ernzerhof, M. Phys. Rev. Lett. 1996, 77, 3865-3868.

(9) Soler, J. M.; Artacho, E.; Gale, J. D.; Garca, A.; Junquera, J.; Ordejón, P. et al. Journal of Physics: Condensed Matter 2002, 14, 2745.

(10) Junquera, J.; Paz, O.; Sánchez-Portal, D.; Artacho, E. Phys. Rev. B 2001, 64, 235111.

(11) Korytár, R.; Pruneda, M.; Junquera, J.; Ordejn, P.; Lorente, N. J. Phys.: Condens. Matter 2010, 22, 385601.

(12) Souza, I.; Marzari, N.; Vanderbilt, D. Phys. Rev. B 2001, 65, 035109.

(13) Mostofi, A. A.; Yates, J. R.; Lee, Y.-S.; Souza, I.; Vanderbilt, D.; Marzari, N. Computer Physics Communications 2008, 178, 685 - 699.

(14) Baldereschi, A.; Baroni, S.; Resta, R. Phys. Rev. Lett. 1988, 61, 734-737.

(15) Wangsness, R. K. Electromagnetic Fields, 2nd Edition, 2nd ed.; Wiley-VCH, 1986.

(16) Press, W. H. Numerical recipes 3rd edition: The art of scientific computing; Cambridge university press, 2007.

(17) Li, Q.; Zou, X.; Liu, M.; Sun, J.; Gao, Y.; Qi, Y. et al. Nano Letters 2015, 15, 58045810.

(18) Roche, S. Phys. Rev. B 1999, 59, 2284-2291.

(19) Torres, L. E. F. F.; Roche, S.; Charlier, J.-C. Introduction to Graphene-Based Nanomaterials; Cambridge University Press: Cambridge, UK, 2014.

(20) Cummings, A. W.; Duong, D. L.; Nguyen, V. L.; Van Tuan, D.; Kotakoski, J.; Barrios Vargas, J. E. et al. Advanced Materials 2014, 26, 5079-5094. 
(21) Kınacı, A.; Haskins, J. B.; Sevik, C.; Çağın, T. Phys. Rev. B 2012, 86, 115410.

(22) Hahn, K. R.; Melis, C.; Colombo, L. Carbon 2016, 96, 429-438. 